\title{
Perfecting the Ultra-violet of Holographic Descriptions of QCD
}

\author{
Nick Evans, Andrew Tedder* \\ School of Physics and Astronomy \\ Southampton University \\ Southampton, SO17 1BJ \\ United Kingdom
}

\begin{abstract}
We investigate imposing a UV cutoff into a simple AdS/QCD model of the rho mesons. The cutoff corresponds to the scale at which QCD moves from perturbative to non-perturbative behaviour, above which the gravity dual will itself become strongly coupled. Simply imposing a cutoff significantly improves the fit to the masses of the tower of excited rho mesons. Formally one should match the couplings of higher dimension operators and the anomalous dimensions of fields to the QCD values at the cutoff. We explore examples of these matchings including looking at the anomalous dimensions of the $\bar{q} \gamma^{\mu} q$ operators and including a $G \operatorname{Tr} F^{4}$ coupling.
\end{abstract}

*evans@phys.soton.ac.uk, ajmt@phys.soton.ac.uk 


\section{Introduction}

The AdS/CFT Correspondence [1, 2] has provided a new holographic methodology for computing in strongly coupled gauge theories. Amongst the properties of QCD-like gauge theories that have been understood in this arena are confinement [3] quarks [4, chiral symmetry breaking [5], and the meson spectrum [7]. Most recently, phenomenological models of QCD in this spirit have been constructed [8]-[14] which agree with QCD meson data at better than the $20 \%$ level.

These models which are classical theories of gravitational and gauge degrees of freedom are all built around asymptotically AdS spaces which extend out to infinite radius. The radial direction in the gravity dual corresponds to energy in the gauge theory. In the rigorous AdS/CFT setting these theories are dual to a conformal strongly coupled gauge theory (usually the $\mathcal{N}=4$ super Yang-Mills theory) with relevant operators. Their return to the strongly coupled fixed point in the UV sustains the weakly coupled gravity description to infinite radius.

In reality a gravitational dual of QCD would only describe the strong coupling regime. Where asymptotic freedom drives QCD to weak coupling, the gravity dual would become strongly coupled itself. One should therefore impose an ultra-violet, large radius, cutoff on the gravity dual. To truly describe QCD one should perform a matching of the gravity dual to QCD in this transition regime in energy; a regime where strong coupling effects might be expected to be setting in already in the field theory. On the gauge side one should match the anomalous dimensions of fields, the couplings of higher dimension operators and also include all operator expectation values. As we pointed out in [15] if all these matchings could be performed one would have a description of QCD in the spirit of perfect lattice actions [17.

This matching seems a hard task, not least because there are potentially an infinite tower of couplings of higher dimension operators possible, but the success of the simple AdS models suggests that deviations from matching to perturbative QCD are not very large. One can hope to follow the path of improved lattice actions and try to identify the most significant deviations from the AdS picture that are needed to better match QCD. One can use QCD data to tune matching parameters and then hopefully further predictions will be more accurate. We previously investigated these ideas [15] in the glueball sector of a pure Yang Mills theory but the paucity of data removed any ability to test the predictions.

Here we will attempt to implement these ideas in the rho meson sector of QCD. There is a reasonable amount of data provided by the masses of the excited states in this sector and the AdS/QCD description [11 is very simple so there are relatively few matching parameters that can be tuned. We will show that just including a UV cutoff makes a substantial improvement to the fit to the meson masses.

We also study how the theory can be brought closer to "perfect" by adjusting the $\bar{q} \gamma^{\mu} q$ operator's anomalous dimension. In addition, we consider the inclusion of a coupling of a 
higher dimension operator in the glue sector of the theory. In both cases a fit to the data suggests these are small effects. Here one is beginning to loose predictiveness again and instead the results are better interpreted in terms of learning about the matching conditions from the data.

\section{$2 \rho$ Mesons in AdS/QCD}

We will use the simple AdS/QCD model of [11] as our starting point. This model has the metric and dilaton

$$
d s^{2}=\left(\frac{d r^{2}}{r^{2}}+r^{2} \eta_{\mu \nu} d x^{\mu} d x^{\nu}\right), \quad \Phi(r)=r^{-2}
$$

The non zero dilaton is present to provide both an IR cut off and to ensure the tower of $\rho$ meson masses grow like $\sqrt{n}$ with $n$ the excitation number. This behaviour is that expected from simple confinement models [12]. The $\rho$ mesons are described by a vector field in this space

$$
S=\int d^{5} x \sqrt{g} e^{-\Phi} \operatorname{Tr}\left(-\frac{1}{4 g_{5}^{2}}\left(F_{L}^{2}+F_{R}^{2}\right)\right)
$$

One seeks normalizable solutions of the linearised equation of motion for $A_{V}^{\mu}$ of the form

$$
A_{V}^{\mu}=f_{n}(r) \rho_{n}^{\mu}(x)=A_{0}^{\mu} f_{n}(r) e^{i k \cdot x}, \quad k^{2}=-M_{n}^{2}
$$

where $A_{V}=\left(A_{L}+A_{R}\right) / 2$ and $A_{0}^{\mu}$ is a constant.

$f_{n}(r)$ must satisfy

$$
\partial_{r}\left(r^{3} e^{-\frac{1}{r^{2}}} \partial_{r} f_{n}\right)+M_{n}^{2} \frac{e^{-\frac{1}{r^{2}}}}{r} f_{n}=0
$$

The normalized solutions have been shown to be

$$
f_{n}=\frac{1}{r^{2}} \sqrt{\frac{2}{1+n}} L_{n}^{(1)}\left(r^{-2}\right)
$$

where $L_{n}^{(m)}$ are the associated Laguerre polynomials. The squared masses are

$$
M_{n}^{2}=4(n+1)
$$

The first five $\rho$ meson excitations predicted by the model (and scaled to the lightest $\rho$ mass) are 


\begin{tabular}{c|c|c}
$n$ & $m_{\rho} / \mathrm{MeV}$ in $\mathrm{QCD}$ & $m_{\rho} / \mathrm{MeV}$ in $\mathrm{AdS} / \mathrm{QCD}$ \\
\hline$\rho$ & 776 & 776 \\
$\rho^{*}$ & 1459 & 1097 \\
$\rho^{* *}$ & 1720 & 1344 \\
$\rho^{* * *}$ & 1900 & 1552 \\
$\rho^{* * * *}$ & 2150 & 1735
\end{tabular}

The observed QCD values are also listed for comparison. Although the $\sqrt{n}$ behaviour is reproduced the masses are consistently low relative to the data (the RMS error, which for $n$ operators $O$ is given by $\epsilon_{R M S}=\left(\sum_{O}\left(\frac{\delta O}{O}\right)^{2} \frac{1}{n}\right)^{1 / 2}$ is $\left.21 \%\right)$.

\section{A UV Cutoff}

\section{$3.1 \rho$ masses}

The first act in regularizing the UV of the theory is to put in a cutoff at large $r$. This cutoff should correspond to the scale at which QCD becomes non-perturbative or, in the holographic dual, the scale below which the classical gravity approximation becomes trustable. We first try to fix the position of this cutoff phenomenologically by varying it's position and computing the $\rho$ meson masses. We look numerically for regular solutions of eqn (44) as described above but now using the UV boundary conditions

$$
f_{n}(r) \sim r^{-2}, \quad f_{n}^{\prime}(r) \sim-2 r^{-3}
$$

at the cutoff rather than at infinite radius. This choice corresponds to setting the dimension of the operator $\bar{q} \gamma^{\mu} q$ to be three at the UV matching scale where QCD starts to become nonperturbative.

We plot the tower of masses in figure 1 as a function of the position of the cutoff $\Lambda$, which we assign both a value of $r$ and the equivalent energy scale in the gauge theory. The $n=0$ state is in each case normalized to the physical $\rho$ mass. The dots represent the experimental values.

Decreasing the cutoff scale from infinite radius raises the masses of the excited tower states as is required to better match the QCD data. Significant corrections only set in when $\Lambda$ is small and the best fit occurs when the cutoff is placed at a radius of $r=0.50$. The fit is then extremely good with an RMS error of $1.8 \%$. The improvement is sufficiently impressive to suggest that imposing a UV cutoff is the appropriate way to improve AdS/QCD.

The value of the QCD strong coupling scale is set by the coefficient in front of the $r^{-2}$ term in the dilaton which explicitly breaks the scale invariance. Throughout we have chosen the coefficient to be one. Matching our results using the $n=0$ state's mass we find that the best fit value of $\Lambda$ corresponds to a mass scale of just $194 \mathrm{MeV}$. Clearly this suggests that the regime of 


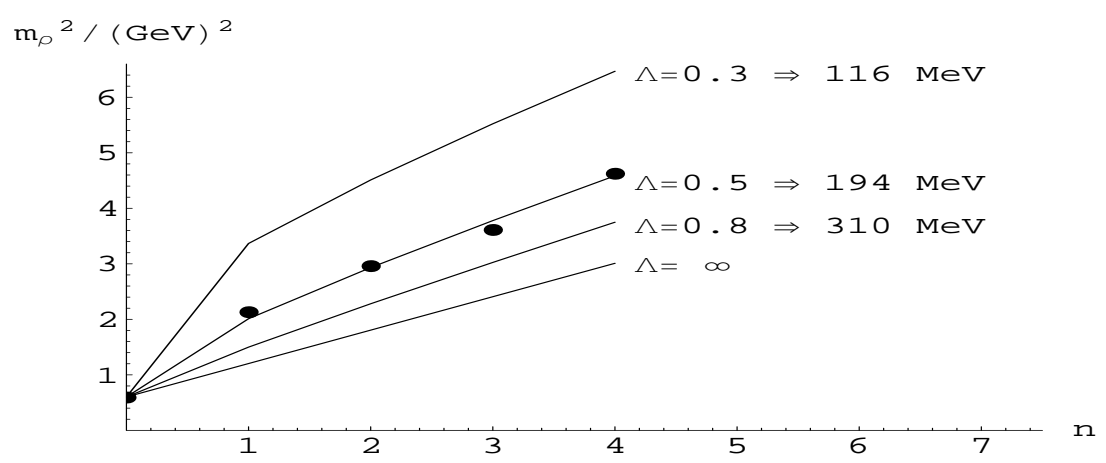

Figure 1: The mass squared of the $n$ lightest $\rho$ meson excitations for different values of the UV cut off listed as both a value of $r$ in the AdS space and the equivalent energy scale. The dots represent the experimental values.

validity of a gravity dual to QCD is actually rather small (the same was found in our previous study of a pure glue theory [15]). Given the precocious asymptotic freedom of QCD perhaps this is not so surprising though. Note the small value of the best fit UV cutoff also means the gravitational theory has a significant dilaton factor throughout it's regime of validity.

It is worth pointing out that with this value of $\Lambda$, we predict the next 3 excited $\rho$ mesons to be at $2320 \mathrm{MeV}, 2475 \mathrm{MeV}$ and $2626 \mathrm{MeV}$. Experimental searches have reached up to 2510 $\mathrm{MeV}$, and so far the highest excited $\rho$ meson found is the final one listed above at $2150 \mathrm{MeV}$.

\subsection{Decay constants}

The $\rho$ meson decay constants can be found by substituting the regular solutions $f_{n}(r) \rho^{\mu}(x)$ back into the $5 \mathrm{~d}$ action and integrating over $r$. The decay constants are given by 8

$$
F_{\rho}^{2}=\frac{1}{g_{5}^{2}}\left(\Lambda^{3} f_{n}^{\prime}(\Lambda)\right)^{2}
$$

Since the large $r$ behaviour of $f_{n}(r) \sim 1 / r^{2}$ for all $n$, the different excited states only differ in their decay constants as a result of the different normalizations of the $f_{n}$. We require that the kinetic terms for the different rho excitations are all canonical which implies imposing

$$
\int_{0}^{\Lambda} d r \frac{e^{-r^{-2}}}{r g_{5}^{2}} f_{n}^{2}=1
$$

In the original AdS/QCD model with the UV cutoff at infinity one finds the decay constants grow as the square root of the excitation number $n$

$$
F_{\rho_{n}}^{2}=\frac{8(n+1)}{g_{5}^{2}}
$$


If one matches $g_{5}$ to the perturbative high energy vector correlator [8, 9] so $g_{5}^{2}=12 \pi^{2} / N_{c}$ then the $n=0 \rho$ has a decay constant $F_{\rho_{0}}^{1 / 2}=260 \mathrm{MeV}$ compared to the physical value of 345 $\mathrm{MeV}$.

In figure 2 we display the results of the same computation with a UV cutoff present. For low cutoffs $F_{\rho_{0}}$ rises: with $\Lambda=194 \mathrm{MeV} F_{\rho_{0}}^{1 / 2}=478 \mathrm{MeV}$. Comparison with the physical value again hints that a low cutoff is appropriate. On the other hand, as the cutoff is brought down the $\sqrt{n}$ behaviour (argued for in [12]) is apparently lost and the higher resonance decay constants fall relative to the $n=0$ case. The reason for this is that the cutoff impedes on the values of $r$ where the wave functions of the eigenstates are substantial. By the time that the cutoff is of order a few hundred $\mathrm{MeV}$ the integral for the normalization is dominated around the cutoff. This makes the computation of the decay constant suspect - formally one needs a description of the physics to higher energies which may lie beyond the region of perturbative validity for the supergravity. Note this contrasts with the computation of the masses - those values are determined by requiring regular solutions in the infra-red away from the cutoff.
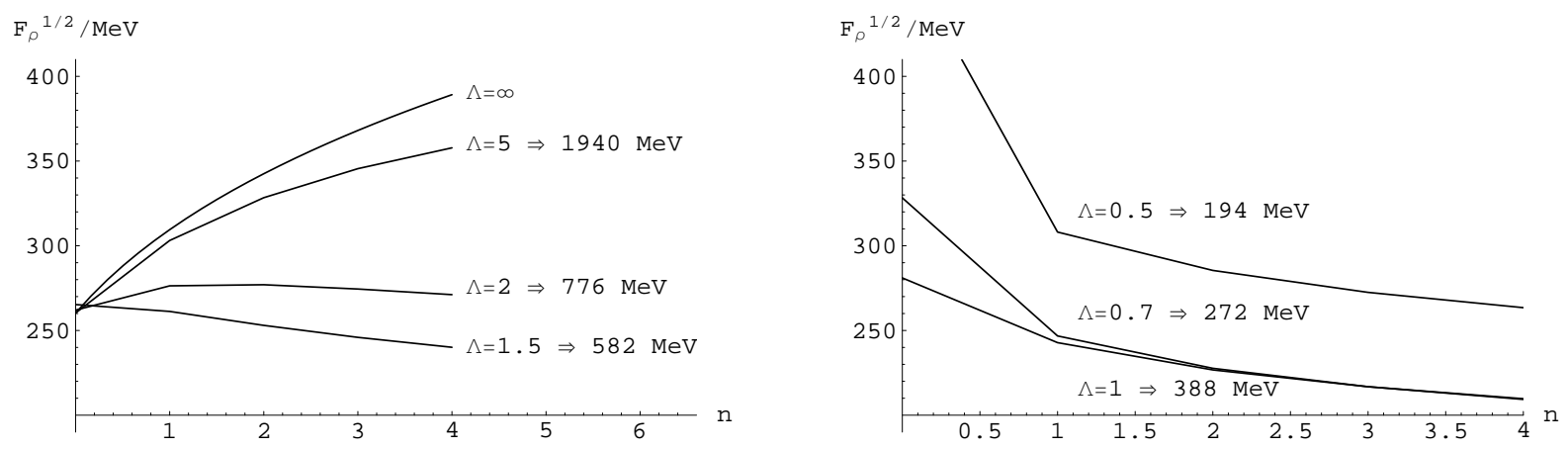

Figure 2: The decay constant $F_{\rho}^{1 / 2}$ for the different $\rho$ excitations plotted for varying UV cut off.

\section{Anomalous Dimensions at the Cutoff Scale}

If one is going to impose a UV cutoff on the gravitational description that lies in the transition region where QCD moves from perturbative to non-perturbative behaviour, one should be careful in matching the theories. It is possible that, for example, substantial non-perturbative effects should be included: in other words, the dimension of the operator $\bar{q} \gamma^{\mu} q$ should not be simply set to three. In the gravity dual this dimension is encoded by the initial conditions set for the eigenfunctions at the cutoff.

One can repeat the computation of the $\rho$ masses using boundary conditions

$$
f_{n}(r) \sim r^{-\omega}, \quad f_{n}^{\prime}(r) \sim-\omega r^{-\omega-1}
$$


at $r=\Lambda$ with $\omega$ a free parameter.

We find that for all values of the cutoff, from $\infty$ down to our best fit value, the preferred value of $\omega$ is two, which is consistent with the naive dimension matching. This appears to tell us that the anomalous dimensions are not large.

However, if one evaluates the derivative of the flows $f_{n}(r)$ at a finite values of $r$ in the case of the model with a UV cut off at infinity then the derivative differs for each value of $n$. This suggests that formally one must allow $\omega$ to vary state by state. Of course this introduces so many free parameters that predictions are lost. It is nevertheless interesting to see how much these anomalous dimensions must be shifted from two to precisely reproduce the observed spectrum. For example for our best fit value of $\Lambda=194 \mathrm{MeV}$ one finds

\begin{tabular}{c|c} 
meson & $\omega$ \\
\hline$\rho^{*}$ & 1.85 \\
$\rho^{* *}$ & 1.98 \\
$\rho^{* * *}$ & 2.12 \\
$\rho^{* * * *}$ & 1.98
\end{tabular}

The corrections are less than $10 \%$.

\section{Coupling of a Higher Dimension Operator}

The presence of large couplings for higher dimension operators before QCD can be matched to the perturbative gravity theory would be another signal of non-perturbative phenomena. There are formally an infinite set of such couplings which might be important. One might hope the couplings of lower dimension operators would grow fastest as one moved into the nonperturabtive regime though. Such couplings, which are irrelevant perturbations, will appear in the gravitational dual as deformations of the metric which grow at large $r$. We know how to encode one simply [15, 16] so will investigate the effect of that.

If we write the metric as

$$
d s^{2}=H^{1 / 2} d r^{2}+H^{-1 / 2} \eta_{\mu \nu} d x^{\mu} d x^{\nu}
$$

Then we can deform the AdS space by allowing it to return to flat space asymptotically,

$$
H(r) \rightarrow r^{-4}+\alpha=r^{-4}\left(1+\alpha r^{4}\right)
$$

The parameter $\alpha$ is a symmetry singlet and has energy dimension -4 . It should therefore be identified with the coupling of the term $G \operatorname{Tr} F^{4}$.

We have repeated the fit to the lightest five $\rho$ meson masses in this deformed geometry. The preferred value of $\alpha$ as a function of $\Lambda$ and the RMS error of the fit are 


\begin{tabular}{cc|c|c}
$\Lambda$ & & $\alpha$ & $\epsilon_{r m s}$ \\
\hline$r=20$ & $(1137 \mathrm{MeV})$ & 0.0011 & $8.18 \%$ \\
$r=10$ & $(5606 \mathrm{MeV})$ & 0.0045 & $7.51 \%$ \\
$r=5$ & $(2718 \mathrm{MeV})$ & 0.019 & $6.77 \%$ \\
$r=1$ & $(469 \mathrm{MeV})$ & 0.75 & $4.70 \%$ \\
$r=0.5$ & $(194 \mathrm{MeV})$ & 0.08 & $1.83 \%$
\end{tabular}

Note $\alpha$ grows as $\Lambda$ is lowered - this is natural since $\alpha$ changes the large $r$ part of the metric. To change the results when only a small $r$ slice of the metric is considered needs a large $\alpha$.

It can be seen that the fit at a given value of $\Lambda$ is improved by the inclusion of $\alpha$. Amusingly as one approaches the best fit value of $\Lambda$ we found above, the preferred value of $\alpha$ suddenly becomes very small. This is a reflection of just how good the fit is from just including a cutoff. We conclude that if an appropriately low cutoff is included the $G \operatorname{Tr} F^{4}$ coupling is in fact a small effect.

\section{Discussion}

A perturbative gravitational dual of QCD should only be expected to work at energies below a few $\mathrm{GeV}$ at best, where QCD is non-perturbative. We have investigated imposing a UV cutoff on an AdS/QCD model of the $\rho$ mesons and found that the data has a fit at the $2 \%$ level with a UV cutoff of a few hundred $\mathrm{MeV}$ (compared to a fit of $21 \%$ with an infinite cutoff). We conclude that the holographic description of QCD should only be used at low energies on a quite small radial interval.

We have also looked at fitting corrections to the anomalous dimension of the operator $\bar{q} \gamma^{\mu} q$ and introducing a coupling of the operator $\operatorname{Tr} F^{4}$. Although these corrections could be used to fine tune the fit by a percent or so they do not appear to be significant corrections to the model. Of course these are only easily implementable examples from an infinite set of possible corrections but finding the corrections to be small provides further understanding of the success of the basic AdS/QCD models. One could also try to include the vacuum expectation values of more operators in the metric (see for example [14]) and a dynamical, predictive mechanism of chiral symmetry breaking [13]. Such effects would be important to study the pion and axial vector meson sectors of the model. As explained in [1] the model used here does not give a good prediction of these sectors because the dilaton form, put in to give the $\sqrt{n}$ rise in masses, does not lead to a sensible condensate prediction. If one attempted to tackle all of these problems then most likely the number of free parameters would rise faster than the number of available data points. Of course this reflects the fact that a perfect action is in the end just a reparametrization of the full QCD spectrum. We hope though that we have identified the imposition of a UV barrier as an important correction and that these other effects are subleading 
in the $\rho$ sector. Putting together a complete model of all sectors including the baryons remains as an important challenge.

\section{References}

[1] J. M. Maldacena, Adv. Theor. Math. Phys. 2, 231 (1998) Int. J. Theor. Phys. 38, 1113 (1999) arXiv:hep-th/9711200; S. S. Gubser, I. R. Klebanov and A. M. Polyakov, Phys. Lett. B 428, 105 (1998) arXiv:hep-th/9802109.

[2] E. Witten, Adv. Theor. Math. Phys. 2, 253 (1998) arXiv:hep-th/9802150.

[3] J. M. Maldacena, Phys. Rev. Lett. 80 (1998) 4859 arXiv:hep-th/9803002; S. J. Rey and J. T. Yee, Eur. Phys. J. C 22 (2001) 379 arXiv:hep-th/9803001.

[4] A. Karch and E. Katz, JHEP 0206 (2002) 043 arXiv:hep-th/0205236; M. Bertolini, P. Di Vecchia, M. Frau, A. Lerda and R. Marotta, Nucl. Phys. B 621 (2002) 157 arXiv:hep-th/0107057; M. Grana and J. Polchinski, Phys. Rev. D 65 (2002) 126005 arXiv:hep-th/0106014.

[5] J. Babington, J. Erdmenger, N. J. Evans, Z. Guralnik and I. Kirsch, Phys. Rev. D 69 (2004) 066007 arXiv:hep-th/0306018; N. J. Evans and J. P. Shock, Phys. Rev. D 70 (2004) 046002 arXiv:hep-th/0403279; N. Evans, J. Shock and T. Waterson, JHEP 0503 (2005) 005 arXiv:hep-th/0502091; M. Kruczenski, D. Mateos, R. C. Myers and D. J. Winters, arXiv:hep-th/0311270; K. Ghoroku and M. Yahiro, Phys. Lett. B 604 (2004) 235 arXiv:hep-th/0408040; T. Sakai and S. Sugimoto, Prog. Theor. Phys. 113 (2005) 843 arXiv:hep-th/0412141; T. Sakai and S. Sugimoto, Prog. Theor. Phys. 114 (2006) 1083 arXiv:hep-th/0507073; E. Antonyan, J. A. Harvey, S. Jensen and D. Kutasov, arXiv:hep-th/0604017; E. Antonyan, J. A. Harvey and D. Kutasov, arXiv:hep-th/0608149; E. Antonyan, J. A. Harvey and D. Kutasov, arXiv:hep-th/0608177;

[6] D. Bak and H. U. Yee, Phys. Rev. D 71 (2005) 046003 arXiv:hep-th/0412170.

[7] M. Kruczenski, D. Mateos, R. C. Myers and D. J. Winters, arXiv:hep-th/0304032; R. C. Myers and R. M. Thomson, arXiv:hep-th/0605017, T. Sakai and J. Sonnenschein, JHEP 0309 (2003) 047, arXiv:hep-th/0305049; J. Erdmenger, J. Große and Z. Guralnik, JHEP 0506 (2005) 052 arXiv:hep-th/0502224; D. Arean and A. V. Ramallo, arXiv:hep-th/0602174, J. Erdmenger and I. Kirsch, JHEP 0412 
(2004) 025 arXiv:hep-th/0408113; R. Casero, A. Paredes and J. Sonnenschein, JHEP 0601 (2006) 127 arXiv:hep-th/0510110; A. Paredes and P. Talavera, Nucl. Phys. B 713 (2005) 438 arXiv:hep-th/0412260; M. Bando, A. Sugamoto and S. Terunuma, arXiv:hep-ph/0602203; J. P. Shock, arXiv:hep-th/0601025; J. Erdmenger, N. Evans and J. Grosse, arXiv:hep-th/0605241.

[8] J. Erlich, E. Katz, D. T. Son and M. A. Stephanov, Phys. Rev. Lett. 95 (2005) 261602 arXiv:hep-ph/0501128.

[9] L. Da Rold and A. Pomarol, Nucl. Phys. B 721 (2005) 79 arXiv:hep-ph/0501218.

[10] G. F. de Teramond and S. J. Brodsky, Phys. Rev. Lett. 94 (2005) 201601 arXiv:hep-th/0501022; S. J. Brodsky and G. F. de Teramond, arXiv:hep-ph/0602252 H. Boschi-Filho and N. R. F. Braga, Eur. Phys. J. C 32 (2004) 529 arXiv:hep-th/0209080; H. Boschi-Filho and N. R. F. Braga, JHEP 0305 (2003) 009 arXiv:hep-th/0212207; S. Hong, S. Yoon and M. J. Strassler, JHEP 0404 (2004) 046 arXiv:hep-th/0312071; L. Da Rold and A. Pomarol, JHEP 0601 (2006) 157 arXiv:hep-ph/0510268; J. P. Shock and F. Wu, arXiv:hep-ph/0603142; K. Ghoroku, N. Maru, M. Tachibana and M. Yahiro, Phys. Lett. B 633 (2006) 602 arXiv:hep-ph/0510334; T. Hambye, B. Hassanain, J. March-Russell and M. Schvellinger, arXiv:hep-ph/0512089.

[11] A. Karch, E. Katz, D. T. Son and M. A. Stephanov, arXiv:hep-ph/0602229.

[12] M. Shifman, arXiv:hep-ph/0507246.

[13] N. Evans and T. Waterson, arXiv:hep-ph/0603249;

[14] C. Csaki and M. Reece, arXiv:hep-ph/0608266.

[15] N. Evans, J. P. Shock and T. Waterson, Phys. Lett. B 622 (2005) 165 arXiv:hep-th/0505250.

[16] N. J. Evans, C. V. Johnson and M. Petrini, JHEP 0205 (2002) 002 arXiv:hep-th/0112058; K. A. Intriligator, Nucl. Phys. B 580 (2000) 99 arXiv:hep-th/9909082; N. R. Constable and R. C. Myers, JHEP 9911 (1999) 020 arXiv:hep-th/9905081.

[17] P. Hasenfratz and F. Niedermayer, Nucl. Phys. B 414 (1994) 785 arXiv:hep-lat/9308004; M. Luscher, Phys. Lett. 158B (1985) 250. 